Relations industrielles

Industrial Relations

\title{
GAP Initiation pratique, par Dominique Lamure, Paris, Collection. L'informathèque, Entreprise moderne d'édition, 1970, 155 pp.
}

\section{Jean-Louis Langevin}

Volume 26, numéro 4, 1971

URI : https://id.erudit.org/iderudit/028284ar

DOI : https://doi.org/10.7202/028284ar

Aller au sommaire du numéro

Éditeur(s)

Département des relations industrielles de l'Université Laval

ISSN

0034-379X (imprimé)

1703-8138 (numérique)

Découvrir la revue

Citer ce compte rendu

Langevin, J.-L. (1971). Compte rendu de [GAP Initiation pratique, par

Dominique Lamure, Paris, Collection. L'informathèque, Entreprise moderne

d'édition, 1970, 155 pp.] Relations industrielles / Industrial Relations, 26(4),

1060-1061. https://doi.org/10.7202/028284ar

Tous droits réservés (C Département des relations industrielles de l'Université Laval, 1971
Ce document est protégé par la loi sur le droit d'auteur. L'utilisation des services d'Érudit (y compris la reproduction) est assujettie à sa politique d'utilisation que vous pouvez consulter en ligne.

https://apropos.erudit.org/fr/usagers/politique-dutilisation/ 
tions, selon le sens de leur évolution politique et des idéologies sociales dominantes, pour se doter d'organismes consultatifs valables.

André ROY

Le Code du travail : sa nature, sa portée, ses effets, par Fernand Morin. Québec, Ministère du travail et de la main-d'oeuvre, 1971, 61 pp.

Ce petit ouvrage n'a aucune prétention scientifique. Il a simplement pour but de mettre à la portée de tous ceux qui sont engagés dans les relations du travail les points essentiels du Code du travail en vigueur actuellement au Québec.

Après une introduction où l'auteur explique brièvement la nature du droit du travail et décrit les principales mesures législatives en ce domaine, l'ouvrage comprend deux chapitres. Le premier traite des «parties en présence dans les rapports collectifs du travail : deux parties bien distinctes; la détermination du syndicat majoritaire $\gg$. Le second chapitre est consacré à «l'élaboration par les parties des conditions de travail: la négociation collective; la convention collective ». Le tout est suivi d'annexes comprenant la liste des lois du travail distribuées selon leur objet, un tableau synoptique du mécanisme prévu au Code du travail, un questionnaire récapitulatif et une brève bibliographie.

Les lecteurs de Relations industrielles connaissent bien l'auteur qui est depuis longtempps un des collaborateurs réguliers. Grâce à sa compétence en droit du travail, à son expérience et à ses grandes qualités de pédagogue, il a réussi à présenter un texte où il a su allier clarté, concision et disposition pour en faire un outil extrêmement utile à tous. On pourra avantageusement s'en servir dans des sessions d'étude à l'intention des travailleurs.

Gérard DION

\section{Dictionnaire des partis communistes et des mouvements révolutionnaires, par François Fejtö, Paris, Casterman, 1971, $238 \mathrm{pp}$.}

Il s'agit non pas d'un dictionnaire mais d'un répertoire par pays des partis, mouvements, organisations considérés comme communistes ou qui se donnent pour telles. Ce répertoire est accompagné quand ils sont disponibles de renseignements sur la date de la fondation, les leaders, les effectifs, la presse et l'orientation idéologique pro-soviétique ou pro-chinoise de ces partis, mouvements et organisations.

Un tel répertoire constitue en soi un instrument de travail utile même en relations industrielles puisqu'il peut se prêter à une étude plus poussée de l'influence que ces divers organisrnes pourraient exercer sur les syndicats du moins lorsqu'il s'agit de pays où le parti communiste n'est pas au pouvoir.

La lecture de ce répertoire confirme l'ampleur du «schisme» qui divise ce monde communiste entre «pro-soviétique » et «pro-chinois».

L'essai d'interprétation de ce schisme contenu dans les premières pages du livre n'est pas non plus sans intérêt. Les divers facteurs de dislocation de l'unité du monde communiste qui ne fut jamais complète même du vivant de Staline, y sont succintement abordés; ce sont le débat idéologique sur l'interprétation du marxisme-léninisme, et la stratégie du mouvement communiste, la remontée spectaculaire des nationalismes, et l'affrontement de l'Union soviéticue et de la Chine pour le contrôle du mouvement communiste international. A cet essai d'interprétation qui n'est qu'esquissé, il manque selon nous une donnée principale soulignée notamment par Raymond Aron: A la politique des blocs de la guerre froide a succédé une politique plus souple, à l'est comme à l'ouest, dès que le développement de l'arme thermonucléaire et de son vecteur principal, la fusée, balistique intercontinental a en quelque sorte dévalorisé l'importance stratégique des états tampons.

\section{Bernard SOLASSE}

GAP initiation pratique, par Dominique Lamure, Paris, Collection L'informathèque, Entreprise moderne d'édition, $1970,155 \mathrm{pp}$.

L'Entreprise moderne d'édition lance une nouvelle collection, l'Informathèque, sous la direction de MM. J.L. Groboillot et J.M. Dethoor du Centre de Calcul de la Chambre de Commerce et d'Industrie de Paris. Cette collection a pour objectif de fournir aux étudiants, 
cadres d'entreprises, fonctionnaires, etc... une initiation à l'outil informatique, ses techniques, son langage et ses diverses utilisations principalement dans le domaine de la gestion.

GAP : initiation pratique est l'un des quatre ouvrages déjà publiés dans la série «Les langages》. GAP (Générateur Automatique des Programmes) est un langage évolué (IBM) utilisé pour les opérations de gestion et principalement pour la comptabilité. Sa principale qualité réside dans son utilisation et sa codification relativement faciles.

L'ouvrage lui-même comporte une série de leçons présentées selon la méthode de l'enseignement programmé. Le lecteur progresse à son propre rythme, grâce aux nombreux tests qui lui permet- tent, à chaque étape, de mesurer son degré de compréhension et de maîtrise du langage. D'accès facile, cet ouvrage nécessité toutefois, à notre avis, une connaissance minimum du fonctionnement «logique » de l'ordinateur. L'apprentissage de tout langage informatique est sans conterdit plus aisé lorsqu'on s'est fait expliquer (démystifier) l'ordinateur.

L'ouvrage de $M$. Lamure constitue donc un manuel de qualité propre à familiariser le dirigeant d'entreprise avec ce langage, précieux outil de gestion. Complété par quelques heures de pratique à l'ordinateur, il assurera à l'etudiant qui le désire, une bonne maîtrise du langage GAP.

Jean-Louis LANGEVIN

\section{POUVOIR ET «POUVOIRS » EN RELATIONS DU TRAVAIL}

Introduction, GÉRARD DION - Pouvoir et «pouvoirs 》 dans les relations du travail, Vincent Lemieux et GÉRARd Dion - Pouvoir et «pouvoirs》 dans l'entreprise privée, Hugues LEYDET - Pouvoir et «pouvoirs» chez l'étatemployeur, JEAN CournoYer - Pouvoir et «pouvoirs d dans les syndicats, Gérard Hébert - Pouvoir et action syndicale, Daniel Vidal - Pouvoir syndical, Bernard SOlasse - Documents de travail.

1 volume, 184 pages - Prix : $\$ 5.50$

LES PRESSES DE L'UNIVERSITÉ LAVAL 